\title{
Reconstruction of spatial distribution of strains in crystals using the energy spectrum of X-ray Moiré patterns
}

\author{
Fodchuk I. M., Balovsyak S. V., Novikov S. M., Yanchuk I. V. and \\ Romankevych V. F.
}

Yuriy Fedkovych Chernivtsi National University, 2 Kotsyubinsky Str., 58002 Chernivtsi, Ukraine ifodchuk@ukr.net

Received: 06.03 .2020

\begin{abstract}
We develop a new approach to the analysis of experimental Moiré patterns obtained with LLL-interferometer. Radial distributions of the energy spectra of Moiré patterns reveal different sensitivities to the sources of local mechanical strains in the low- and high-frequency ranges. This offers new possibilities for determining the magnitudes of total mechanical strains and reconstructing their spatial distributions in crystals.
\end{abstract}

Keywords: LLL-interferometers, Moiré patterns, strain fields, frequencies of radial distributions, Fourier energy spectra

UDC: $548.4-548.734$

\section{Introduction}

Optical interferometric methods have given great impulse to novel techniques of multicrystalline interferometry of X-ray and neutron radiations [1-3]. The first studies [2, 4] have shown that the $\mathrm{X}$-ray interferometry reveals extremely high sensitivity to any disturbance of crystalline structure $[5,6]$. Currently, this method has many important practical applications in metrology, e.g. when determining the Avogadro constant [3, 7, 8] and standardizing X-ray wavelengths [3]. It also represents a powerful tool for the phase tomography studies of medical and biological samples [9-11]. Implementation of combined optical and X-ray interferometries in Refs. [12,13] has discovered even such a fine effect as small influence of surface pressure on the interplanar distances in silicon crystals. A promising setup for a multicrystalline interferometer has been suggested in the work [14].

The effect of structural defects and external actions on the formation of Moiré images using a three-crystal LLL-interferometer has been studied in Refs. [15-19]. At the same time, it is worthwhile that the quality of interference images is influenced significantly by the structural defects and macroscopic mechanical strains present in the plates introduced in interferometer. Since the X-ray interferometry rests upon basic laws of optics [20], the studies of formation of $\mathrm{X}$-ray interference patterns in the analyzer of a so-called LLL-interferometer, which creates a predetermined strain field, are important for the further development of methods aimed at reproducing the strain-field distributions and calculating the strain-tensor components created by defects [21].

Nowadays the dynamic theory of X-rays enables one to carry out any necessary numerical simulations of the Moiré patterns under conditions of predetermined strain fields [22-25]. If one analyzes some experimental Moiré patterns, the approach makes it potentially possible to obtain all the quantitative information about the sources of residual or active strains and their spatial distribution. The same refers to the nature of appropriate effects [24]. The models that involve 
different distributed sets of concentrated forces, which have been used in the works [16, 19, 22-25], allow for investigating the influence of long-period or localized strain fields on the X-ray diffraction and the interference processes occurring in LLL-interferometers.

The Moiré images created by one-dimensional sets of local concentrated forces on the output surface of the analyzer of three-crystal LLL-interferometer have been investigated in the works $[23,24]$ for the both cases of orientations of those forces parallel or perpendicular to the diffraction vector. Different sets of force magnitudes and their spatial distributions selected in the above works have testified qualitative agreement of theoretical Moiré patterns with the experimental ones, which have been obtained from the residual strain fields created by the scratches during scribing the output surface of the analyzer [15].

New strain-induced Moiré bands occur near the places where the concentrated forces are localized, i.e. in a transition region where the strains change their signs. The shape of those bands reflects a general displacement field of the atomic planes created near the sources of forces [24]. On the other hand, increase in the period of the Moiré bands upon withdrawal of the concentrated forces reflects the behaviour of the total strain field.

The presence of a constant phase shift in one of the interfering beams in the interferometer analyzer induces a decrease in the period, the contrast and the size of the area where the straininduced Moiré bands are formed [23]. The area of efficient interaction of the phase and strain Moiré components depends not only on the value of constant phase shift, but also on the magnitude and distribution of local force sources.

In the study [25] we have developed the approach that can reproduce dependence of the mean spatial frequency of the energy spectra of Moiré images on the magnitude of strain field generated as a total of local strain sources. This enables one to estimate the magnitude of the averaged residual strain, which has generated the Moiré pattern in the process of scribing the analyzer surface, using the average spatial frequency of the energy spectrum [15]. At the same time, one could not establish the relation between the parameters of energy spectra and the spatial configuration of strain distribution.

In the present study, we consider new approaches to the analysis of Moiré intensity distributions basing on the Fourier energy spectrum method [25, 26]. We demonstrate different sensitivities to the spatial distributions of local strain sources, which are revealed by the radial energy spectra of Moiré images in their low-frequency and high-frequency ranges. As a consequence, one can determine on this basis both the total magnitude of the strains and their spatial distribution in crystals.

\section{Method for determining the distribution of force sources $\boldsymbol{P}_{\boldsymbol{n}}$}

To calculate Moiré intensity distribution as a function of magnitude of the concentrated forces and their spatial arrangement, we have utilized the algorithm and the analytical relations given in Refs. [22-25]. A scheme of action of some local strain sources $P_{1}, \ldots, P_{N}$ on the output surface of the LLL-interferometer is shown in Fig. 1. A sum $P_{N L}$ presented in relative units corresponds to the sum of forces $P_{d}$ (in Newtons). For example, we have $P_{d}=0.08 \mathrm{~N}$ in the case of $P_{N L}=100$, which characterizes a region of weak strains [15]. The relationship for the sum of forces $P_{N L}$ and the $P_{d}$ parameter is described as (see Ref. [22])

$$
P_{d}=\sin \theta \frac{P_{N L}}{2 \pi G L}|\vec{H}|,
$$

where $\theta$ is the Bragg angle of X-ray reflection (e.g., for the (220) $\mathrm{CuK}_{\alpha}$-radiation), $G$ the shear modulus, $\vec{H}$ the diffraction vector, and $L$ the length of a line segment along which a series of forces $P_{1}, \ldots, P_{N}$ acts. 


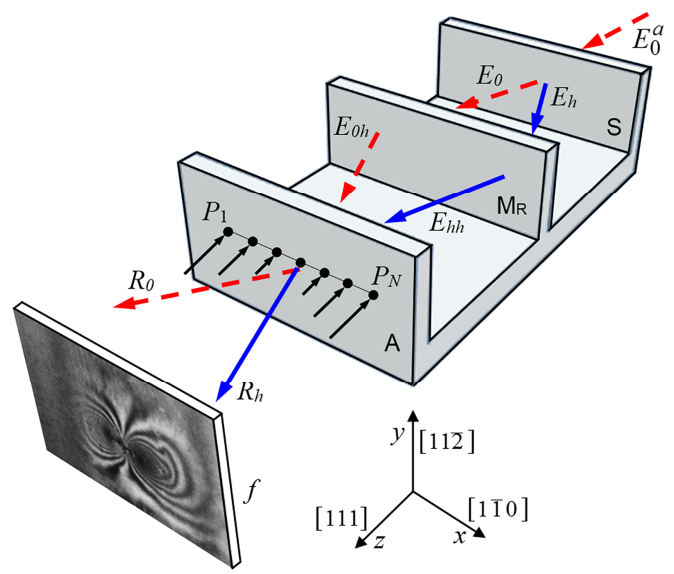

Fig. 1. Scheme of X-ray LLL-interferometer (see also Ref. [1]): $\mathrm{S}$ - splitter, $\mathrm{MR}$ - mirror, $\mathrm{A}$ - analyzer, $E_{0}^{a}-$ incident X-ray beam, $E_{0}$ and $E_{\mathrm{h}}$ - refracted and diffracted beams after splitter $S, E_{0 \mathrm{~h}}$ and $E_{\mathrm{hh}}$ - diffracted beams after mirror MR, and $R_{h}$ and $R_{0}$ - output beams from the interferometer, which form a Moiré pattern $f$ due to the action of concentrated forces $P_{1}, \ldots, P_{N}$.

In order to establish the relationship between the strain and the Moiré pattern parameters, we have performed the appropriate calculations for five different $P_{N L}$ values $(21,52,84,105$ and 147). This has been done for the following three characteristic shapes $S_{i}$ of the distributions $P_{n}: S_{1}-\mathrm{a}$ uniform distribution $P_{n}^{1}, S_{2}$ - a parabolically curved distribution $P_{n}^{2}$ with a minimum force value corresponding to the centre, and $S_{3}$ - a curved distribution $P_{n}^{3}$ with a maximum in the centre [18]. This area of $P_{N L}$ values reflects completely the initial and intermediate stages of formation of additional Moiré bands in Fig. 2.
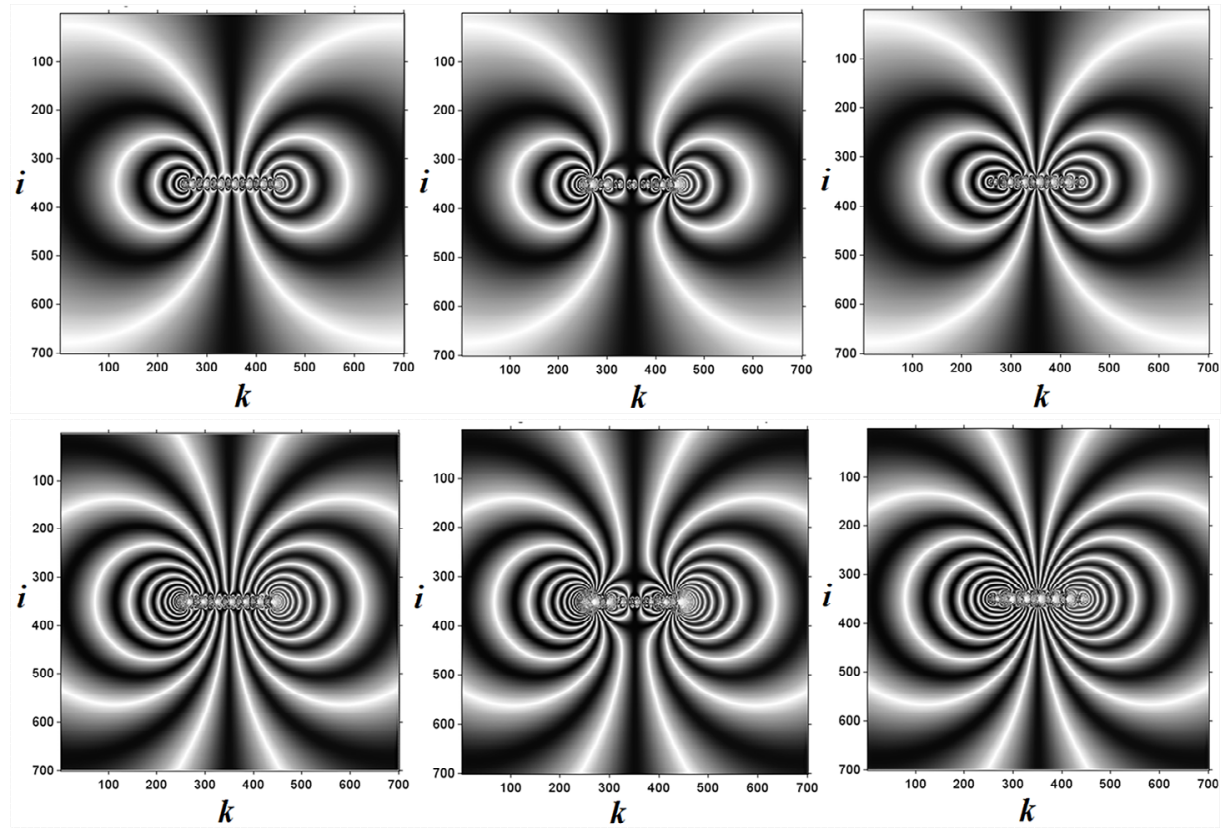

(a)

(b)

Fig. 2. Fragment of a set of X-ray Moiré patterns, which are presented as matrices $f(i, k)$ for the distributions of concentrated forces $P_{n}^{1}, P_{n}^{2}$ and $P_{n}^{3}$ (with the shapes $S_{1}, S_{2}$ and $S_{3}$, respectively). $P_{N L}$ is equal to 52 (panel a) and 105 (panel b). 
To carry out Fourier transformation and calculate the energy spectra, the Moiré patterns $R_{h}(x, y)$ shown in Fig. 2 have been represented as digital matrixes $f=f(i, k)$, where the index $i$ concerns the series $1, \ldots, M_{i}$ and $k$ the series $1, \ldots, N_{k}$. Here $M_{i}$ is the height and $N_{k}$ the width of the pattern in pixels.

The X-ray Moiré patterns $f$ depend significantly on the magnitude and the type of local force distribution (see Fig. 2). We are to note that solving uniquely the inverse problem (i.e., reconstructing spatial distribution and magnitudes of the strains that generate a Moiré pattern, issuing from this pattern) is still hindered by essential difficulties. We offer an original method to overcome them and solve the problem.

For this aim, we perform a two-dimensional direct discrete fast Fourier transform of the matrix $f(i, k)$. The energy spectrum $P_{S}$ (or the spectral power density) is then determined by the square module of $F_{C}[26]$ :

$$
P_{S}=\left|F_{C}\right|^{2} \text {. }
$$

The radial distribution (or profile) $P_{R}(d)$ of the Moiré pattern is calculated using the energy spectrum $P_{S}$. Here $d$ is the radial frequency number $\left(d=1, \ldots, N_{R}\right)$ and $N_{R}=\min \left(M_{i}, N_{k}\right) / 2$.

In any Moiré pattern, a high-frequency noise is present in the most of cases [27]. It manifests itself as a background in the radial distributions $P_{R}(d)$, especially at high spatial frequencies $\left(v_{r}>0.2 \mathrm{pel}^{-1}\right)$. Therefore one should remove the noise component from the distributions $P_{R}$ in order to calculate accurately the average spatial frequency $\bar{v}_{R}$. This spatial frequency is calculated from the radial distribution $P_{R}(d)$ of the energy spectrum [25] as follows:

$$
\bar{v}_{R}=\frac{\sum_{d=2}^{N_{R}} P_{R}(d) \cdot v_{r}(d)}{\sum_{d=2}^{N_{R}} P_{R}(d)} .
$$

Here the constant component $P_{R}(1)$ is not taken into account when calculating the average frequency. Note that, contrary to the $P_{R}$ values for a given frequency, the average spatial frequency $\bar{v}_{R}$ of the radial distribution $P_{R}$ does not depend on the experimental conditions under which the image has been obtained.

Our analysis of the Moiré patterns calculated for the cases of different sums of forces $P_{N L}$ enables finding a correlation between the $\bar{v}_{R}$ and $P_{N L}$ parameters. This makes it possible to reconstruct the sums of the concentrated forces $P_{N L}^{r}$ on the basis of the frequency $\bar{v}_{R}$, using a piecewise linear fitting [25]. However, no correlation has been found between the frequency $\bar{v}_{R}$ and the nature of the force distribution $P_{n}$. As a result, there is a need in examining the correlation between the distributions $P_{n}$ and $P_{R}$.

We develop a theoretical approach to calculate the distribution of forces $P_{n}$, issuing from the parameters of Moiré patterns. For this aim, we have analyzed a set of reference images shown in Fig. 2. The relationship between the nature of $P_{n}$ and the frequency of radial distribution $P_{R}$ can be ascertained after analyzing the shape of $P_{R}$ in different ranges of the $v_{r}$ frequency. We have performed the analysis for the dependence $P_{R}\left(v_{r}\right)$ not only for the distributions $P_{R}$ containing highfrequency oscillations, but also for the distributions $P_{R p}$, which are obtained after transforming $P_{R}$ to the logarithmic scale and fitting it with a polynomial of $20^{\text {th }}$ degree (see Fig. 3). 


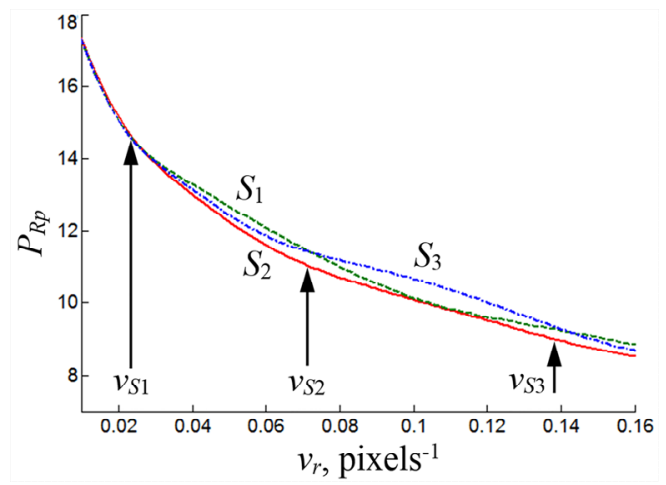

Fig. 3. Fragments of fitted radial distributions $P_{R p}\left(v_{r}\right)$ of the energy spectra $P_{S}$ of Moiré patterns on a semi-log scale. Frequencies $v_{S 1}, v_{S 2}$ and $v_{S 3}$ are the boundaries of different ranges, $P_{N L}=105$, and $S_{1}, S_{2}$ and $S_{3}$ are the shapes of force distributions.

It has been demonstrated that the distributions $P_{R p}\left(v_{r}\right)$ exhibit different sensitivities to the nature of force distribution in the two alternative frequency ranges (see Fig. 3). Here the range \#1 is limited by the spatial frequencies $v_{S I}$ and $v_{S 2}$, while the range \#2 is limited by the frequencies $v_{S 2}$ and $v_{S 3}$. The frequencies $v_{S 1}, v_{S 2}$ and $v_{S 3}$, which are the abscissas of intersection points of the distributions $P_{R p}$ with the shapes $S_{1}$ and $S_{3}$, have been determined for each sum of forces $P_{N L}$ separately (see Fig. 3).

Fig. 4 shows dependences of the range boundaries $v_{S 1}, v_{S 2}$ and $v_{S 3}$ on the sum of forces $P_{N L}$, which have been obtained for a whole set of reference Moiré patterns. Using a piecewise linear fitting, such dependences can be used to calculate the frequencies $v_{S 1}, v_{S 2}$ and $v_{S 3}$ for random $P_{N L}$ values. Then the relative areas $S_{R 1}$ and $S_{R 2}$ can be estimated for the radial distribution $P_{R}\left(v_{r}\right)$ inside the calculated frequency ranges $\left(v_{S 1}, v_{S 2}\right)$ and $\left(v_{S 2}, v_{S 3}\right)$ :

$$
\begin{aligned}
& S_{R 1}=\sum_{v_{r}=v_{S 1}}^{v_{S 2}} P_{R}\left(v_{r}\right) / \sum_{v_{r}=v_{S 1}}^{v_{S 3}} P_{R}\left(v_{r}\right), \\
& S_{R 2}=\sum_{v_{r}=v_{S 2}}^{v_{S 3}} P_{R}\left(v_{r}\right) / \sum_{v_{r}=v_{S 1}}^{v_{S 3}} P_{R}\left(v_{r}\right) .
\end{aligned}
$$

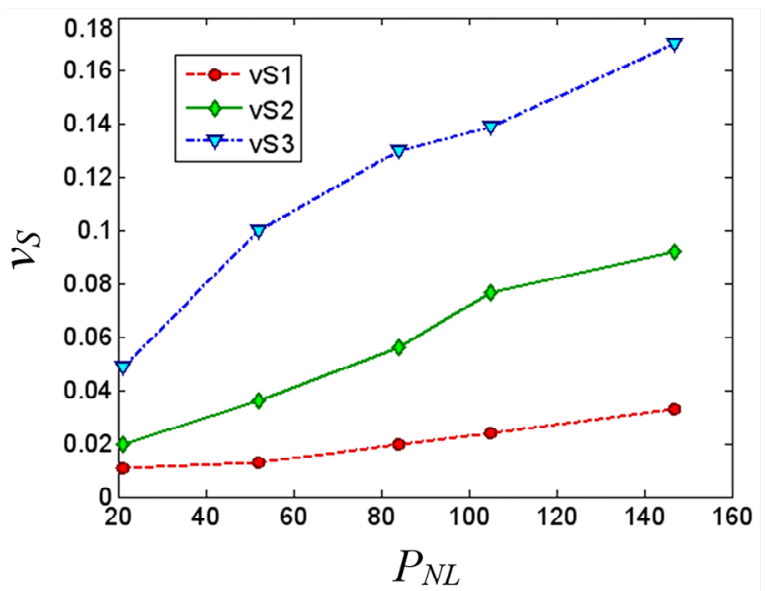

Fig. 4. Dependences $v_{S 1}\left(P_{N L}\right), v_{S 2}\left(P_{N L}\right)$ and $v_{S 3}\left(P_{N L}\right)$ for the boundaries of frequency ranges, as calculated for the Moiré patterns shown in Fig. 2. 
The $S_{R 1}$ and $S_{R 2}$ values have been averaged for all the Moiré patterns with the same sum value $P_{N L}$. The result is given by the average areas $S_{A 1}$ and $S_{A 2}$. The approximate mean areas $S_{A 1}^{a}$ and $S_{A 2}^{a}$ have been calculated by piecewise linear fitting of the dependences $S_{A 1}\left(P_{N L}\right)$ and $S_{A 2}\left(P_{N L}\right)$, basing on the calculated $P_{N L}$ values. The normalized areas $S_{N 1}$ and $S_{N 2}$ (for $P_{R}$ ) are then determined by the equations

$$
S_{N 1}=\frac{S_{P 1}}{S_{A 1}^{a}}, S_{N 2}=\frac{S_{P 2}}{S_{A 2}^{a}} .
$$

A transformation of the areas from $S_{R 1}$ and $S_{R 2}$ to $S_{N 1}$ and $S_{N 2}$ suggested above yields in the fact that the values $S_{N 1}$ and $S_{N 2}$ are no longer dependent on the force sum $P_{N L}$ but depend mainly on the shape of the force distribution $P_{n}$.

The values $S_{N 1}$ and $S_{N 2}$ have been averaged for the reference Moiré patterns corresponding to the same force distribution $\left(S_{1}, S_{2}\right.$ or $\left.S_{3}\right)$, thus resulting in the corresponding mean normalized values $S_{P 1}$ and $S_{P 2}$ for each of the force distributions. The force distributions $S_{1}, S_{2}$ and $S_{3}$ correspond to the theoretical amplitudes of sine function $A_{P}$, which are equal respectively to $0,-0.7$ and 0.7 . However, the dependences obtained thus far are still ambiguous and do not allow one to calculate $A_{P}$ following from $S_{P 1}$ or $S_{P 2}$ (see Table 1).

Table 1. $S_{P 1}, S_{P 2}$ and $S_{P 3}$ values depending on $P_{R}$ as derived for the set of images in Fig. 3.

\begin{tabular}{ccccc}
\hline $\begin{array}{c}\text { Shape of forces } \\
\text { distribution }\end{array}$ & $A_{P}$ & $S_{P 1}$ & $S_{P 2}$ & $S_{P 3}$ \\
\hline$S_{2}$ & -0.7 & 1.0224 & 0.8622 & 0.9612 \\
$S_{1}$ & 0 & 1.1110 & 0.8208 & 1.0000 \\
$S_{3}$ & 0.7 & 1.0237 & 1.2904 & 1.1256 \\
\hline
\end{tabular}

This is why we have calculated the parameter $S_{P 3}$ from Table 1, which is determined as a linear combination of $S_{P 1}$ and $S_{P 2}$ using the proportion of gold cross section. In this manner we obtain a unique dependence of $A_{P}$ on the parameters of energy spectrum of the Moiré pattern:

$$
S_{P 3}=S_{P 1} \cdot 0.618+S_{P 2} \cdot 0.382
$$

Eq. (7) implies the following conditions: (i) if $S_{P 3}=1$, then there is a uniform shape $S_{1}$ of force distribution $\left(A_{P}=0\right)$, (ii) if $S_{P 3}<1$, we have the shape $S_{2}$ with a minimum at the centre $\left(A_{P}<0\right)$ and, finally, (iii) the inequality $S_{P 3}>1$ means the shape $S_{3}$ with a maximum at the centre $\left(A_{P}>0\right)$.

The final empirical dependence of the reconstructed amplitude $A_{P r}$ of sine function, which describes the force distribution $P_{n}$ (see Fig. 5), on the parameter $S_{P 3}$ of the energy spectrum is as follows:

$$
A_{\mathrm{Pr}}=2\left(\frac{1}{1+\exp \left(-\left(S_{P 3}-1\right) \cdot C_{1}\right)}-\frac{1}{2}\right),
$$

It is described according to the $S_{P 3}\left(A_{P}\right)$ values (see Table 1). In our case we have $C_{1}=43$ (at $S_{P 3} \leq 1$ ) or $C_{1}=14$ (at $S_{P 3}>1$ ). The dependence $A_{P r}\left(S_{P 3}\right)$ provides the $A_{P r}$ changes occurring in the acceptable range (from -1 to 1 ) for arbitrary $S_{P 3}$ values. The dependences $A_{P r}\left(S_{P 3}\right)$ are different for the force distributions with the shapes $S_{2}$ and $S_{3}$, which is taken into account by different $C_{1}$ coefficients. 


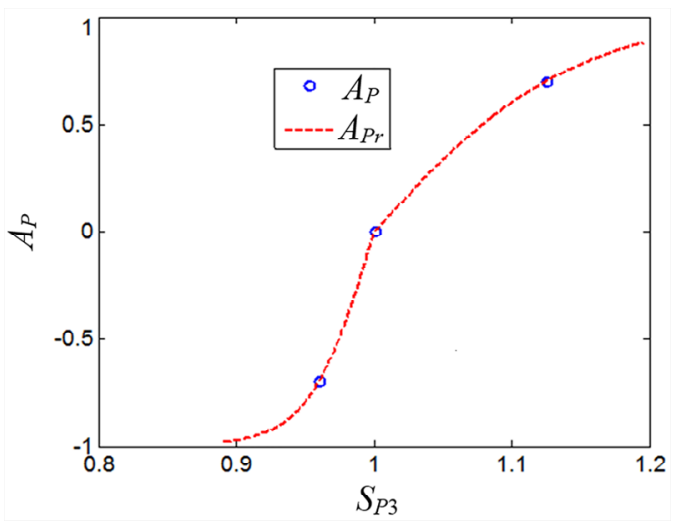

Fig. 5. Dependence $A_{P r}\left(S_{P 3}\right): S_{P 3}$ is the average normalized area of the radial distributions $P_{R}$ for the images shown in Fig. 2, the parameters $A_{P}$ are given by Table 1, and $A_{P r}$ is the amplitude value reconstructed using Eq. (8), which describes the force distribution $P_{n}$.

\section{Comparison of calculated and experimental Moiré patterns}

Our approach suggests a solution to the inverse problem of finding the sum $P_{N L}$ of forces and the force distribution $P_{n}$ (with $n=1, \ldots, N$ ), following from the analysis of Moiré patterns. The appropriate algorithm is as follows. First, the total force $P_{N L}^{r}$ is determined from the mean frequencies $\bar{v}_{R}$ of the radial distribution $P_{R}$ [18] (see Fig. 6). The mean square error of $P_{N L}^{r}$ calculations is negligible for the set of reference images: we obtain the absolute error $R_{P L}=1.4$ and the relative error $\varepsilon_{P L}=1.7 \%$.

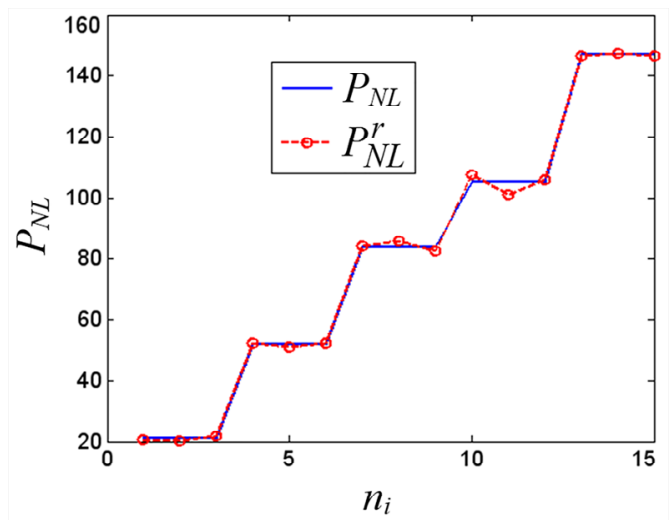

Fig. 6. Comparison of experimentally reconstructed $\left(P_{N L}^{r}\right)$ and theoretical $\left(P_{N L}\right)$ forces: the $P_{N L}^{r}$ values found from the $\bar{v}_{R}$ values coincide in practice with the $P_{N L}$ values that correspond to the images $n_{i}$ (see Fig. 2). The cases $P_{N L}=21,52,84,105$ and 147 are considered.

The force distribution $P_{n}$ (with $n=1, \ldots, N$ ) can be determined issuing from the analysis of $P_{R}$ shape. The force values $P_{n}$ (of which sum equals to $P_{N L}$ ) are described by the sine function with the amplitude $A_{P r}$ and the period $T_{P}=2 N-1$, where the amplitude $A_{P r}$ can be calculated basing on the $P_{R}$ values and Eq. (8).

As a result, Fig. 7 shows the force distributions $P_{n r}$ reconstructed using the analysis of Moire patterns depicted in Fig. 2. The root mean square error, i.e. the discrepancy between the $P_{n r}$ and $P_{n t}$ values remains acceptable for all of the reference images. Namely, we have the absolute error $R_{P}=1.5$ and the relative error $\varepsilon_{P}=1.8 \%$. Different panels of Fig. 7 illustrate the following specific cases: 
(a) the shape $S_{3}, P_{N L}^{r}=105.3$, and $A_{P r}=0.66$; (b) the shape $S_{1}, P_{N L}^{r}=107$, and $A_{P r}=0.09$; (c) the shape $S_{2}, \quad P_{N L}^{r}=102.4$, and $A_{P r}=-0.63$; (d) a new shape similar to $S_{2}, P_{N L}^{r}=100.7$, and $A_{P r}=-0.99$. Here $n$ is the number of concentrated forces. In particular, the relative $P_{n r}$ error is $\varepsilon_{P}=1.6 \%$ for the reference images (see panels a, b and $\mathrm{c}$ in Fig. 7) and it becomes only slightly higher $\left(\varepsilon_{P}=3.1 \%\right)$ for the test image of Fig. $7 \mathrm{~d}$.
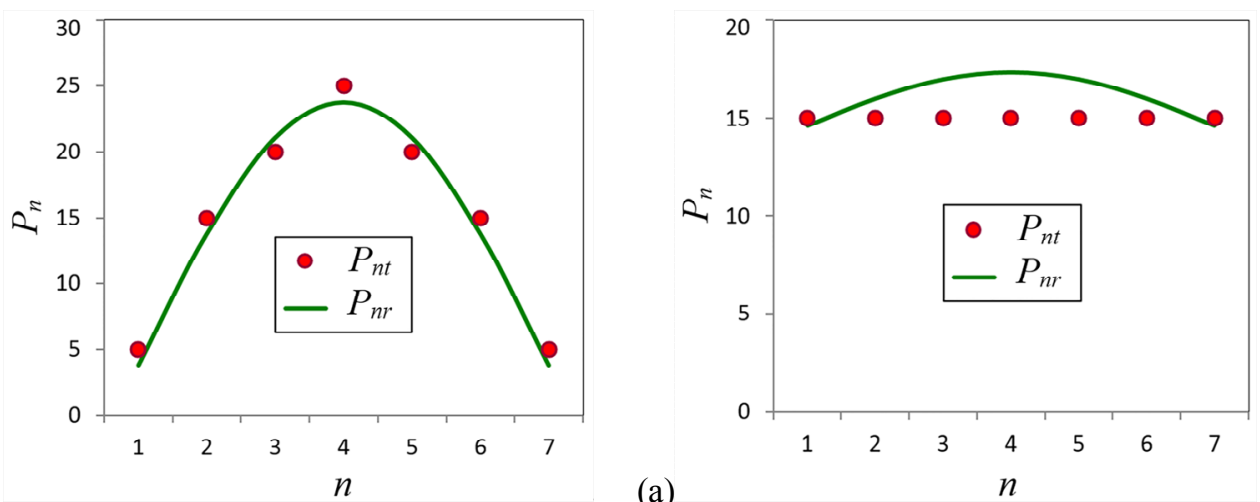

(b)
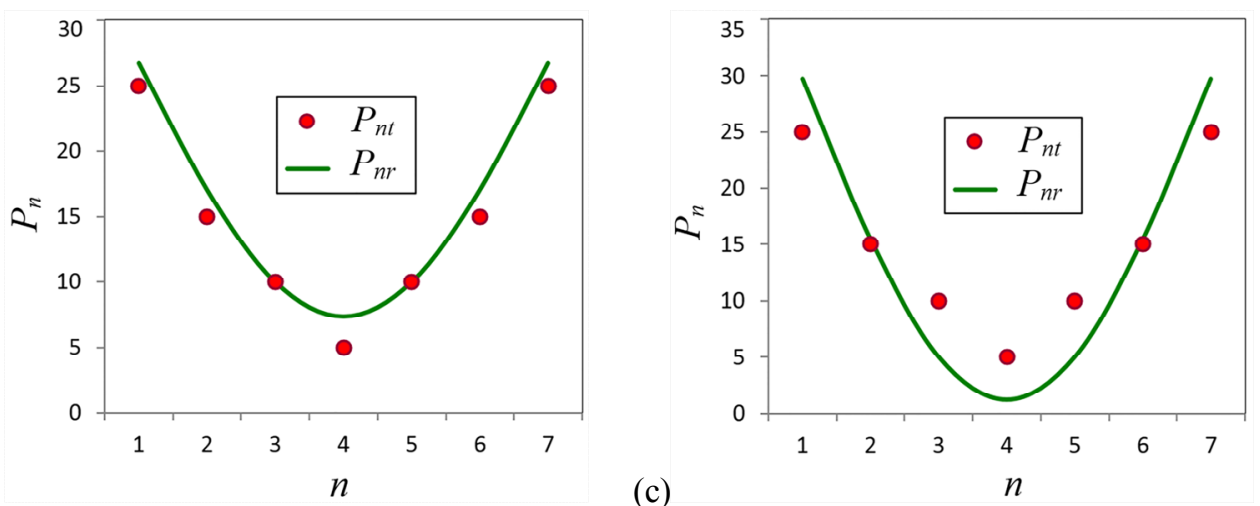

Fig. 7. Theoretical $\left(P_{n t}\right)$ and reconstructed $\left(P_{n r}\right)$ force distributions as functional dependences. The distributions are obtained basing on both the reference Moiré patterns (see Fig. $2 \mathrm{~b}$ ) and the test Moiré pattern for the cases of $P_{N L}=105$ and various shapes $\left(S_{1}, S_{2}\right.$ and $\left.S_{3}\right)$.

It is important that a satisfactory agreement between the reconstructed $\left(P_{n r}\right)$ and theoretical $\left(P_{n t}\right)$ forces has been obtained not only for the reference images and the distribution shapes $S_{1}, S_{2}$ and $S_{3}$, but also for the test image with a new distribution shape (see Fig. 7d). The root mean square deviation between the $P_{n r}$ and $P_{n t}$ values for the test image $\left(P_{N L}=21, \ldots, 147\right)$ is quite acceptable: the absolute error amounts to $R_{P}=2.3$ and the relative error to $\varepsilon_{P}=2.7 \%$. This confirms the fact that our method is versatile when reconstructing the force distributions with various shapes.

Finally, we have estimated a sum of residual forces $P_{N L}^{r}$ and a possible force distribution $P_{n r}$ (see Fig. 8b), using our method and analyzing the experimental Moiré pattern obtained in Ref. [15] (see Fig. 8a). The obtained sum of forces, $P_{N L}^{r}=43.7$, is close to the data derived with the independent method [25] $\left(P_{N L}=41.7\right)$, while the relative calculation error for the sum of forces is equal to $\varepsilon_{P L}=4.6 \%$. This testifies correctness and great potentials of our approach to the quantitative analysis of Moiré patterns. 

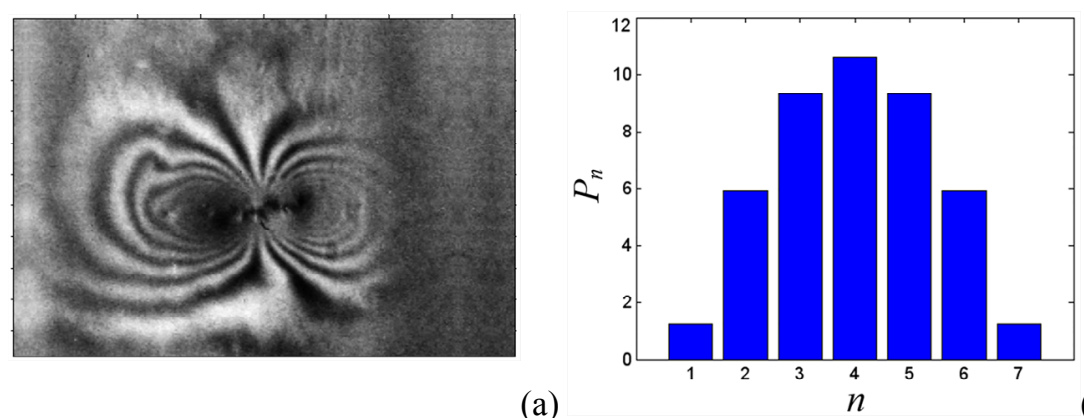

(a)

Fig. 8. (a) Experimental Moiré pattern $(800 \times 541$ pel) caused by residual strains resulted from an indenter on the output surface of LLL-interferometer analyzer [15]; (b) reconstructed force distribution (profile) that causes these strains and results in the Moiré pattern shown in Fig. 8a. The parameters thus obtained are $P_{N L}^{r}=43.7$, $P_{d}=0.035 \mathrm{~N}$ and $A_{P r}=0.75$.

\section{Conclusions}

Summarizing the main results of the present work, we state the following. The X-ray Moiré intensity distributions depend essentially on both the magnitude and the nature of spatial distribution of local forces acting on the surface of LLL-interferometer analyzer. In particular, we have revealed that the $P_{R}\left(v_{r}\right)$ dependence shows different sensitivities to the nature of force distribution in the low-frequency and high-frequency ranges. The approach to analysis of the Moiré patterns suggested by us allows determining the total magnitude of active forces and, moreover, reconstructing their spatial distribution.

The total force $P_{N L}$ that causes a particular energy spectrum $f$ can be determined from the mean spatial radial frequency $\bar{v}_{R}$ by fitting the dependences $P_{N L}\left(\bar{v}_{R}\right)$. The relative error for calculating the $P_{N L}$ parameter is acceptable for both the reference $\left(\varepsilon_{P L}=1.7 \%\right)$ and experimental $\left(\varepsilon_{P L}=4.6 \%\right)$ Moiré patterns.

The spatial distribution of the acting forces $P_{n r}$ can be determined from the analysis of the shape of $P_{R}$ using the energy spectrum $f$ and taking into account the total force $P_{N L}$. The shape of the spatial distribution $P_{n r}$ is functionally described by sinusoids, whose amplitudes can be calculated following from the normalized areas of distributions $P_{R}$ for the two alternative frequency ranges. The relationship between the force distribution $P_{n r}$ and the parameter $P_{R}$ has been found after analyzing a set of reference Moiré patterns.

The method for reconstructing the spatial distributions of concentrated forces $P_{n r}$ suggested in this work can be used to find random forces and corresponding mechanical strains in crystals, which are spatially localized along a linear segment and of which values can be fitted by the sine function.

\section{References}

1. Bonse U and Hart M, 1965. X-Ray interferometer. Appl. Phys. Lett. 6: 155-156.

2. Bonse U, Graeff $\mathrm{W}$ and Materlik G, 1976. X-Ray interferometry and lattice parameter investigation. Rev. Phys. Appl. (Paris). 11: 83-87.

3. Bonse U, Uebbing H, Bartcher M and Nubhardt M, 1994. X-Ray and Neutron interferometry and the measurement of fundamental constants. J. Metrolog. 31: 195-201.

4. Deslattes R D, 1969. Optical and X-ray interferometry of silicon lattice spacing. Appl. Phys. Lett. 15: 386-388. 
5. Cristiansen G, Gerward L and Andersen A L, 1971. A study of the strain field of grown-in dislocations in a silicon X-ray interferometer. J. Appl. Cryst. 4: 370-375.

6. Hart M, 1972. A complete determination of dislocation Burgers vectors by X-Ray interferometry. Phil. Mag. 26: 821-831.

7. Deslattes R D, Henins A, Bowman H A, Schoonover R M, Carroll C L, Barnes I L, Machlan L A, Moore L J, and Shields W R, 1974. Determination of the Avogadro constant. Phys. Rev. Lett. 33: 463-466.

8. Becker P, 2001. History and progress in the accurate determination of the Avogadro constant. Rep. Prog. Phys. 64: 1945-2008.

9. Momose A, Kawamoto S and Koyama I, 2003. Demonstration of X-ray Talbot interferometry. Japan. J. Appl. Phys. 42: 866-868.

10. Momose A, Takeda T and Itai Y, 1996. Phase-contrast X-ray computed tomography for observing biological specimens and organic materials. Rev. Sci. Instrum. 66: 1434-1436.

11. Momose A, 2005. Recent advances in X-ray phase imaging. Jap. J. Appl. Phys. 44: 63556367.

12. Ferroglio L, Mana G and Massa E, 2008. Si lattice parameter measurement by centimeter Xray interferometry. Opt. Express. 16: 16877-16888.

13. Massa E, Mana G and Kuetgens U, 2009. Comparison of the INRIM and PTB lattice-spacing standards. J. Metrologia. 46: 249-253.

14. Snigirev A, Snigireva I, Kohn V and Yukin V, 2009. X-ray nanointerferometer based on Si refractive bilenses. Phys. Rev. Lett. 103: 064801.

15. Raransky M D, Shafranuk V P and Fodchuk I M, 1985. X-ray interferometric image of deformation fields around dislocation clusters. Metallofizika. 7: 63-71.

16. Raransky M D, Fodchuk I M, Sergeev V N and Gimchinsky O G, 1993. The influence of ultrasonic deformations on the moiré images. Metallofizika. 15: 72-79.

17. Raransky N D, Struk Ja M, Fodchuk I M, Shafranuk V P and Raransky A N, 1995. Applied possibilities of an X-ray diffraction interferometry. Proc. SPIE. 2647: 457-467.

18. Fodchuk I M, Raransky N D and Struk Ya M, 2002. The Moiré images of defects in X-ray three-crystal LLL-interferometry. Metallophysics and Advanced Technologies. 24: 617-628.

19. Fodchuk I M, Raransky N D and Struk Ya M, 2002. X-ray LLL-interferometry of crystals deformed by concentrated force. Ukr. J. Phys. 47: 1057-1064.

20. Gevers R, 1962. Dynamical theory of moiré fringe patterns. Phil. Mag. 7: 1681-1720.

21. Authier A. Dynamical theory of X-ray diffraction. Oxford: Science Publications (2001).

22. Fodchuk I M and Raransky M D, 2003. Moiré images simulation of strains in X-ray interferometry. J. Phys. D: Appl. Phys. 36: A55-A60.

23. Fodchuk I M, Novikov S M and Yaremchuk I V, 2016. Direct and inverse problems in X-ray three-crystal LLL-interferometry. Appl. Opt. 55: B120-B125.

24. Fodchuk I M, Novikov S M and Yaremchuk I V, 2016. Reconstruction of a residual strain field in a crystal-analyser of a LLL-interferometer. Metallophysics and Advanced Technologies. 38: 389-403.

25. Balovsiak S V, Novikov S M, Fodchuk I M and Yaremchuk I V, 2019. Analysis of moiré $\mathrm{X}$-ray images of deformed crystals using radial distribution of the Fourier energy spectrum. Metallophysics and Advanced Technologies. 41: 389-402.

26. Gonzalez R and Woods R. Digital image processing. Upper Saddle River: Prentice Hall (2002). 
27. Kosarevych R J, Rusyn B P, Korniy V V and Kerod T I, 2015. Image segmentation based on the evaluation of the tendency of image elements to form clusters with the help of point field characteristics. Cybernetics and Systems Analysis. 51: 704-713.

Fodchuk I. M., Balovsyak S. V., Novikov S. M., Yanchuk I. V. and Romankevych V. F. 2020. Reconstruction of spatial distribution of strains in crystals using the energy spectrum of X-ray Moiré patterns. Ukr.J.Phys.Opt. 21: 141 - 151. doi: 10.3116/16091833/21/3/141/2020

Анотація. Розвинуто новий підхід до аналізу експериментальних муарових картин, одержаних за допомогою LLL-інтерферометра. Радіальні розподіли енергетичних спектрів муарових зображень виявляють різну чутливість до джерел локальних механічних напружень у низькочастотному та високочастотному діапазонах. Це пропонує нові можливості для визначення загальної величини механічних деформачій та реконструкиії їхнього просторового розподілу в кристалах. 